\title{
Large solar-type magnetic reconnection model for magnetar giant flare
}

\author{
Youhei Masada \\ Institute of Astronomy and Astrophysics, Academia Sinica \\ email:masada@asiaa.sinica.edu.tw
}

\begin{abstract}
We construct a magnetic reconnection model for magnetar giant flare in the framework of solar flare/coronal mass ejection theory. As is the case with the solar flare, the explosive magnetic reconnection plays a crucial role in the energetics of the magnetar flare. A key physics controlling the energy transport in the system, on the other hand, is the radiative process unlike that in the solar flare. After the release of the magnetic energy via the magnetic reconnection, the radiative heat flux drives the baryonic evaporation. Our model can predict that the baryonic matter evaporated in the preflare stage would be the origin of the radio emitting ejecta observed in association with the giant flare on 2004 December 27 from SGR1806-20.
\end{abstract}

Keywords. Gamma rays: bursts - stars: individual (SGR 1806-20) - MHD - stars: neutron

\section{Introduction}

There has recently been growing evidence that soft gamma-ray repeaters (SGRs) is the population of strongly magnetized neutron star, so called Magnetar (Thompson \& Duncan 1995). Activities in this object are believed to be powered by the dissipation of strong magnetic fields. Here we focus on the giant flare with enormous energy and long bursting duration which is observed from SGRs.

The giant flare from SGR1806-20 is the most recent and energetic event. It is characterized by an ultra-luminous hard spike with the energy $10^{46} \mathrm{erg}$, which decays rapidly into a soft pulsating tail. A preflare activity is additionally detected before the main burst. The exceptional phenomenon observed in association with the flare is the radio emitting ejecta loaded by the baryonic matter of $10^{24} \mathrm{~g}$. The origin of the baryon-load is, however, not understood in the context of existing model (Thompson \& Duncan 1995).

Analogous mass ejection events are observed accompanying with solar flare, that is called coronal mass ejection (CME). Observational features of the solar flare/CME are similar to those of the magnetar flare (Lyutikov 2006). The solar flare/CME theory is applied for constructing an alternative model for the magnetar giant flare.

\section{Magnetic Reconnection Model}

In the magnetar flare, the balance between the reconnection heating and the radiative cooling gives the flare temperature unlike the solar flare controlled by the conductive cooling (Shibata \& Yokoyama 1999). Considering that the blackbody cooling retains the thermal balance of the system, the balance equation is, with the flare duration $\Delta t$; $E_{\mathrm{GF}}=\left(4 \pi R^{2} \sigma_{B} T_{\mathrm{f}}^{4}\right) \Delta t$, where $E_{\mathrm{GF}}=\left(B^{2} / 4 \pi\right) R L^{2}$ is the reconnection-induced energy.

After the flare begins, the released energy is transported to the magnetar crust by the photon flux. Then the incident radiative heat flux should counterbalance with the outgoing enthalpy flux of the evaporation flow. The baryon number density of the evaporation flow $n_{\mathrm{ev}}$ is thus obtained from the balance equation; $F_{\mathrm{GF}} \simeq\left(n_{\mathrm{ev}} k_{B} T_{\mathrm{f}}\right) v_{\mathrm{ev}}$, where 


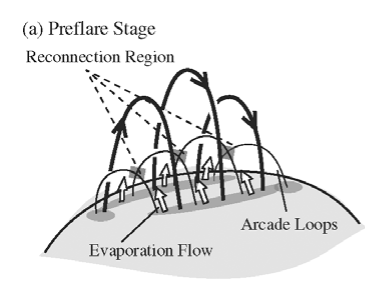

(b) Quiescent Stage

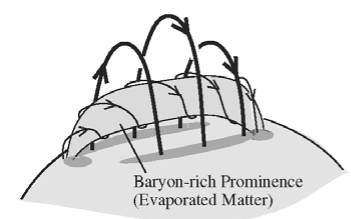

(c) Main Burst Stage

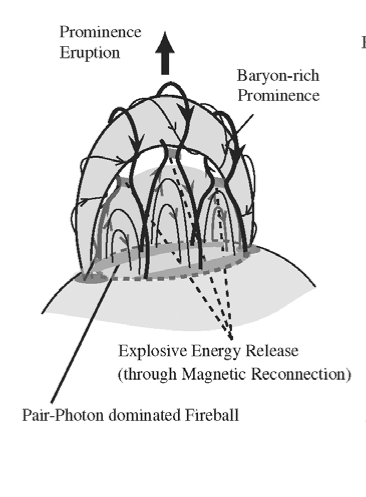

(d) Mass Ejection Stage

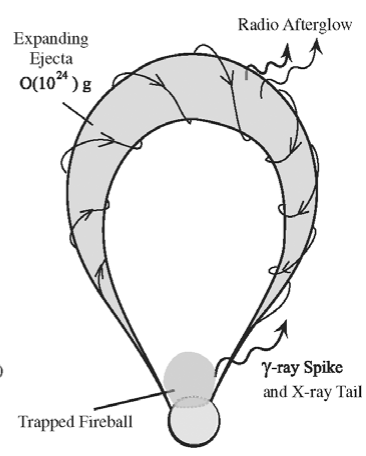

Figure 1. Solar-type Magnetic Reconnection Model for Magnetar Giant Flare

$F_{\mathrm{GF}}=E_{\mathrm{GF}} /\left(4 \pi R^{2} \Delta t\right)$ is the radiative heat flux, $v_{\mathrm{ev}}$ is the velocity of the evaporation flow. For given parameters $E_{\mathrm{GF}}, \Delta t$ and $R$, we can obtain $T_{\mathrm{f}}, v_{\mathrm{ev}}$, and $n_{\mathrm{ev}}$ by solving these equations. The evaporated mass can be thus given by a simple scaling relation,

$$
M_{\mathrm{ev}}=3.4 \times 10^{24}\left(R / 10^{6} \mathrm{~cm}\right)^{2}\left(T_{\mathrm{f}} / 10^{8} \mathrm{~K}\right)^{3}(\Delta t / 1.0 \mathrm{sec}) .
$$

Our model suggest that the preflare stage with $T_{\mathrm{f}} \simeq 10^{8} \mathrm{~K}$ and $\Delta t \simeq 1.0 \mathrm{sec}$ is suitable for suppling the baryonic matter loaded in the radio emitting ejecta observed with the giant flare from SGR1806-20 (see Masada et al. 2008 for more details).

\section{A Possible Scenario for the Giant Flare from SGR 1806-20}

In the preflare stage, the magnetic energy stored in the active region is released via the magnetic reconnection. The radiative heat flux transports the released energy and heats the magnetar crust up. Then the pressure of the crustal matter increases drastically and drives the upward evaporation flow. A hot and dense prominence trapped by the post-flare loop is created by the evaporated matter with the mass $M_{\mathrm{ev}} \simeq 10^{24} \mathrm{~g}$.

After the preflare stage, the prominence eruption causes global field reconfiguration and triggers main burst. The released energy in the main burst stage is converted into the kinetic energy of the erupting matter and the radiative energy of the post-flare loop. The ejected matter would be observed as the radio emitter. The remained post-flare loop can be the origin of the trapped fireball, and which produces the luminous $\gamma$-ray spike and the subsequent hard X-ray pulsating tail.

Our proposed scenario for the Magnetar giant flare is summarized in Figure 1 schematically. An important prediction from our model is that the preflare activity would be the origin of the baryonic matter loaded in the radio emitting ejecta. This suggest that the radio afterglow is expected to be observed only in a giant flare with preflare activity.

\section{References}

Lyutikov, M. 2006, MNRAS 367, 1594

Masada, Y., Nagataki, S., Shibata, K., \& Terasawa, T. 2008, arXiv:0803.3818

Shibata, K. \& Yokoyama, T. 1999, ApJL 526, L49

Thompson, C. \& Duncan, R. C. 1995, MNRAS 275, 255 